\title{
Reply to A. G. C. A. Meesters et al.'s comment on "Biotic pump of atmospheric moisture as driver of the hydrological cycle on land"
}

\author{
A. M. Makarieva and V. G. Gorshkov \\ Petersburg Nuclear Physics Institute, Gatchina, St. Petersburg, Russia \\ Received: 24 April 2009 - Revised: 20 July 2009 - Accepted: 20 July 2009 - Published: 24 July 2009
}

\begin{abstract}
Condensation removes water vapor molecules from the gas phase and reduces the weight of the air column. This disturbs hydrostatic equilibrium and makes air circulate under the action of the recently described evaporative force. Meesters, Dolman and Bruijnzeel (2009) criticized the physical bases of the new circulation driver with a major claim that the ascending air motions induced by the evaporative force should rapidly restore the hydrostatic equilibrium and become extinguished. Here we respond that in fact these air motions sustain the disequilibrium of air pressure through the reduction of the weight of the air column via condensation that continuously occurs as the ascending moist air cools. In the traditional meteorological paradigm condensation is primarily considered in terms of the effect it has, via latent heat release, on air density, while its immediate effect on the weight of air column is not accounted for. The critique of Meesters et al. is therefore informative in highlighting the traditional lines of thought that should be re-visited to incorporate the new physical knowledge. Such an effort is arguably worthy of undertaking as the evaporative force concept bears tangible potential for solving some of the key problems that are challenging modern atmospheric science.
\end{abstract}

\section{Introduction: the key concern of Meesters, Dolman and Bruijnzeel (2009)}

Meesters, Dolman and Bruijnzeel (2009) (hereafter MDB) criticized the physical concept of the evaporative force proposed by Makarieva and Gorshkov (2007) (hereafter MG). We will briefly introduce the reader to the problem.

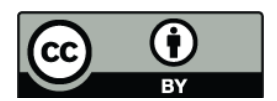

Correspondence to: A. M. Makarieva (elba@peterlink.ru)
In hydrostatic equilibrium air pressure at any height is equal to the weight of the air column above that height. The weight of an air column is equal to the sum of weights of air components. Air pressure is the sum of partial pressures of air components. Thus, when partial pressure of each component is equal to the weight of that component ("component equilibrium" in terms of MDB, "aerostatic equilibrium" in terms of MG), air as a whole is in hydrostatic equilibrium ("bulk equilibrium" in terms of MDB).

In the presence of a sufficiently large vertical lapse rate of air temperature the air column above height $z$ becomes too cold to "bear a sufficient amount of water vapor for its weight to compensate saturated water vapor partial pressure at height $z "$ (MG, p. 1020). Condensation removes water vapor from the air column and creates a strong disequilibrium of the vertical distribution of water vapor that is compressed by more than six times compared to its componentequilibrium distribution. Not affecting the non-condensable dry air components, condensation thus disturbs the hydrostatic equilibrium of air as a whole, so that the only possible stationary stable state of the moist atmosphere becomes the dynamic state when air circulates under the action of the evaporative force. MDB realize that the motion is indeed induced, because obviously "disequilibrium for one air component cannot coexist for long with equilibrium of the other components, since that would mean ... bulk disequilibrium, and hence the initiation of restoring motion" (MDB, Sec. 3.1).

The key concern of MDB is therefore that the motion induced by the evaporative force rapidly extinguishes after it restores the hydrostatic equilibrium of air as a whole. This claim is supported by reference to "common experience" (MDB, Sect. 3.2). In this response we demonstrate that the same process (condensation) that creates the componentdisequilibrium of water vapor also sustains the bulk disequilibrium of air as a whole, so that the resulting motion is not

Published by Copernicus Publications on behalf of the European Geosciences Union. 
transient but stably persists as long as there is water vapor in the air column to sustain continuous condensation. Hydrostatic equilibrium of air as a whole never exists in the atmosphere saturated with water vapor.

\section{The disturbing effect of condensation}

Let us first have a brief look on how condensation is treated in the traditional meteorological paradigm. As an air parcel ascends adiabatically, it expands at the expense of thermal energy of air molecules, so that its temperature drops. If the air parcel is saturated with water vapor, condensation occurs and latent heat is released. This reduces the rate at which the temperature of the lifting parcel drops. Accordingly, the saturated moist adiabatic lapse rate $\Gamma_{m}\left(\Gamma_{m} \sim 5 \mathrm{~K} \mathrm{~km}^{-1}\right.$ at $T=288 \mathrm{~K}$ ) is sufficiently smaller that the dry adiabatic lapse rate $\Gamma_{d}=9.8 \mathrm{~K} \mathrm{~km}^{-1}$. Warmer air has lower density than colder air at the same pressure (not at the same height as incorrectly stated by MDB in Sect. 4). Air column saturated with water vapor is considered to be unstable with respect to the appearance of macroscopic motions if the lapse rate of air temperature $\Gamma$ is larger than $\Gamma_{m}$. In this case, if an air parcel is occasionally displaced adiabatically in the upward direction, its temperature becomes higher and density lower than that of the environment, and the parcel will move upward governed by the acquired positive buoyancy. A saturated air column with $\Gamma=\Gamma_{m}$ is considered to be neutral with respect to such buoyancy-driven convection. In this case an occasional displacement of the saturated air parcel is not expected to bring about a difference in temperature and, hence, density between the air in the parcel and the environment, so the initial displacement should not disturb hydrostatic equilibrium of the air column and should not induce vertical motion. As demonstrated below, in contrast to this traditional knowledge, hydrostatic equilibrium is never preserved in the air column saturated with water vapor; such an air column is always unstable with respect to the appearance of macroscopic motions.

Weight is the product of mass and acceleration of gravity. Mass of an air column is equal to the number of air molecules in the column multiplied by their molecular masses. When the number of air molecules in the column is preserved, its weight remains unchanged and independent of density. Heating of the air column that reduces air density via temperature increase does not change the weight of the air column. In contrast, condensation changes the number of gas molecules in the air column and instantaneously reduces the weight of the air column irrespective of the effects it might have on air density. Thus, when the air parcel saturated with water vapor is occasionally displaced upward in the presumably neutral environment with $\Gamma=\Gamma_{m}$, some of its water vapor is removed from the gas phase. The weight of the air column where the displacement took place diminishes. The initial bulk air pressure in the lower atmospheric levels no longer equals the bulk weight of the air column. The initial hydrostatic equilibrium of air as a whole (MDB's bulk equilibium) is disturbed and the accelerating upward motion is initiated in the air column. This upward motion of expanding and cooling moist air sustains the continuous process of condensation and does not allow the hydrostatic equilibrium of air as a whole to set in. The motion continues as long as there is water vapor in the rising air to sustain condensation. The key processes of air cooling and condensation were ignored in the thought experiment of MDB intended to illustrate the presumed "restoration" of bulk equilibrium (Sect. 3.2).

Preservation of the unrealistic hydrostatic equilibrium of air as a whole, where the component-disequilibrium shortage of water vapor in the upper atmosphere is compensated by the component-disequilibrium excess of dry air (Fig. 1 of MDB), would imply that the disappearance of water vapor molecules from the air column would have to be compensated by the appearance of dry air molecules. However, there are no processes that would produce dry air molecules from nothing in the upper atmosphere so that their weight precisely compensated the weight of water vapor molecules that are removed by condensation. The bulk hydrostatic equilibrium of MDB does not exist.

Condensation will always occur in the ascending saturated air parcel if the rate of temperature decrease within the parcel exceeds the critical value of $\Gamma_{\mathrm{H}_{2} \mathrm{O}}=1.2 \mathrm{~K} \mathrm{~km}^{-1}$ (MG, Eq. 12). Since $\Gamma_{m}$ is larger than $\Gamma_{\mathrm{H}_{2} \mathrm{O}}$, this means that the air column saturated with water vapor is always convectively unstable and there will always be macroscopic air motions present in such a column. Importantly, these are motions driven not by buoyancy differences considered in the traditional paradigm, but by the direct disturbance of hydrostatic equilibrium of air as a whole by condensation.

Confusing the effects condensation and heating have on air weight and density, MDB speak of these processes in a misleading manner. It is stated that "the removal of water from the vapor phase" during condensation "acts in the opposite direction" to the process of local expansion of the air caused by latent heat release (MDB, Sect. 4). However, the reduction of air weight caused by condensation cannot be to any degree "opposed" by whatever changes of air density, as these are independent physical processes. (If one is in a possession of one kilogram of some gas and someone steals five grams from that store, the gas owner will be left with 995 grams of the gas irrespective of how he/she would attempt to heat/cool/expand/compress the remaining amount "to oppose" the loss.) The neglect of the critical effect of condensation on the weight of air column by MDB does not appear to be an oversight of particular authors, but an inherent feature of the traditional meteorological paradigm. The critique of MDB is valuable in bringing to light this important problem. 


\section{Other concerns of MDB}

Concerns of MDB expressed in Sect. 3.2 bear more to one's general acquaintance with the bases of atmospheric circulation (driven by whatever force) rather than specifically to the evaporative force physics. In particular, MDB do not understand why "the strong force predicted by the original equations to be acting upon the vertical column" should be "redistributed over a flow trajectory that includes a long horizontal stretch"; they expect the hurricane velocities of about $50 \mathrm{~m} \mathrm{~s}^{-1}$ to be "common above any evaporating surface"; and they have no idea of how vertical velocity $w_{f}=5.6 \mathrm{~mm} \mathrm{~s}^{-1}$ (MG, p. 1026) is derived.

To resolve these concerns, one should first recall that atmospheric air circulates. Any physical process making air move should therefore provide power for the motion along a close trajectory, i.e. for the ascending and descending air motions as well as for the horizontal motions that occur in the opposite directions in the upper and lower parts of the air column. Accordingly, there appears a non-equilibrium pressure difference along each of the four (two vertical and two horizontal) circulation legs. Consider, for example, the effect of differential heating. When two horizontally adjacent vertical air columns with initially equal surface air pressures $p_{0}$ are instantaneously warmed to different temperatures $T_{1}>T_{2}$, this causes air in the first column to expand in the upward direction, so that the resulting air pressure $p_{1}(z)=p_{0} \exp \left(-z / h_{1}\right)\left(h_{1}=R T_{1} / M g, R\right.$ is the universal gas constant, $M$ is air molar mass, $g$ is acceleration of gravity) becomes larger than air pressure in the second, colder, column at the same height at any height $z>0$. This does not, however, mean that the differential heating will move the whole warmer column in the horizontal direction along this pressure gradient. Instead, the initial air motions are presumed to redistribute the non-equilibrium pressure difference along the four circulation legs in such a manner that air moves upward in the warmer region, downward in the colder region, horizontally from the colder to the warmer region at the lower, and in the opposite direction in the upper, part of the air column. Similarly, action of the evaporative force working in the upward direction leads to a redistribution of the non-equilibrium air pressure difference $\Delta p \sim p_{v}$, where $p_{v}$ is the characteristic magnitude of water vapor partial pressure, along both vertical and horizontal (MG, p. 1022) parts of the streamline, see Makarieva and Gorshkov (2009) for more details. Note that in the case of differential heating the disturbance of hydrostatic equilibrium in the ascending and descending circulation legs is not created by the horizontal temperature difference per se but appears only after air motion is induced and the air mass is horizontally redistributed. In the case of the evaporative force the hydrostatic equilibrium is instantaneously broken by condensation, the air mass is reduced (mass non-conservation) and then the air motion is induced by this disturbance.
Second, the atmosphere is exceedingly wider than high, such as in the majority of circulation patterns the horizontal part $L$ of the streamline is much larger than its vertical part $h$. Horizontal air flow goes through a narrower vertical cross-section of area $\sim h L$ compared to vertical air flow that goes through a much broader horizontal cross-section of area $\sim L^{2}$. To sustain mass balance of the flowing air, horizontal velocities $u$ at $h \ll L$ are always larger than vertical velocities $w$, with the approximate integral continuity equation for air being $u h=w L$ (MG, p. 1025). (Similarly, water flows more rapidly through narrow than through broad parts of the tube.) Therefore, maximum velocities $w_{\max } \sim 50 \mathrm{~m} \mathrm{~s}^{-1}$ are produced by the evaporative force in the vertical direction only in very compact circulation events such as tornadoes (where $h \sim L$ ), while in hurricanes, where $h \ll L$, these maximum velocities are developed in the horizontal plane. Note also that at $L \gg h$ the uniform distribution of the non-equilibrium pressure difference $\Delta p \sim p_{v}$ over the length of the entire streamline makes most part of this difference fall on the horizontal part of the streamline, while the non-equilibrium pressure difference in the vertical dimension (where air ascends and descends) becomes much less than $p_{v} h / L$. This does not imply any violation of Dalton's law (MG, p. 1022), see also Feynman et al. (1963, Chap. 40, Sect. 1), because Dalton's law pertains to the static equilibrium of gas mixture and does not constrain partial pressure distributions of air components when air is circulating.

Finally, one should take into account the effect of surface friction. When most part of the power of the evaporative force is spent to increase the kinetic energy of moving air, maximum velocities are produced. When a considerable part of this power is spent to overcome surface friction (which contribution is proportional to length of the horizontal part of the streamline), the resulting stationary velocities are obviously smaller. Accordingly, vertical velocity $w_{\max }$ in Eq. (18) of MG is obtained, with no account of friction, for a compact circulation event $h \sim L$ where the vertical disequilibrium air pressure difference is of the order of $p_{v}$, while vertical velocity $w_{f}=5.6 \mathrm{~mm} \mathrm{~s}^{-1}$ is obtained for a horizontally extensive stationary circulation with $h \ll L$ and taking surface friction into account by equating the powers of friction force and the evaporative force (MG, p. 1026). (A more detailed consideration of friction is given by Makarieva and Gorshkov (2009). Note also that the power of the evaporative force is proportional to vertical velocity and coincides with the evaporation power only in large-scale stationary circulation patterns.) In summary, $w_{f}$ is smaller than $w_{\max }$ due to the different geometry and friction effects in the considered circulation patterns driven by the evaporative force.

Here it is pertinent to discuss the "lifting velocity" of $0.4 \mathrm{~mm} \mathrm{~s}^{-1}$ obtained by MDB in Sect. 3.1 by a procedure referred to as "a simple calculation". The details of this calculation are not revealed, which makes it difficult to pinpoint exactly where MDB err when obtaining this physically untenable result. MDB claim that such a velocity corresponds 
to evaporation rate of $E=1 \mathrm{~mm} \mathrm{~h}^{-1}$, which means that water vapor is added to the atmospheric column at a rate of $E_{v}=0.27 \mathrm{~g} \mathrm{~m}^{-2} \mathrm{~s}^{-1}$. Water vapor is transported away from the surface layer by the ascending air at a rate of $\rho_{v} w$, where $w$ is vertical velocity and $\rho_{v}$ is water vapor density (and by eddy diffusion at a similar rate). Not to allow for the oversaturation of water vapor at the surface the balance $E_{v} \sim \rho_{v} w$ should be observed. However, at $w=0.4 \mathrm{~mm} \mathrm{~s}^{-1}$ of MDB this balance implies $\rho_{v}=0.7 \mathrm{~kg} \mathrm{~m}^{-3}$ (cf. air density $\rho \sim 1 \mathrm{~kg} \mathrm{~m}^{-3}$ ). Such a high density of water vapor is only possible at temperatures $T \sim 90{ }^{\circ} \mathrm{C}$ and not at $T=15^{\circ} \mathrm{C}$ indicated by MDB for their calculation. More importantly, without taking the resistance forces into account it is not at all possible to obtain a stationary value of vertical velocity. Adding water vapor to a volume occupied by air one performs work against air pressure. In the simplest case, when power $P$ of this process is constant and spent on the increment of kinetic energy $K=m w^{2} / 2$ of a constant mass $m$ of air, $P=d K / d t=m w d w / d t$, it is easy to see that the air will accelerate in a complex manner with acceleration $a \equiv d w / d t=P / m w$ declining with growing velocity from the initial high values.

MDB do not specify to what degree the "simple calculation" that yielded the physically implausible air velocity was based on the "traditional" theory of evaporation mentioned in Sect. 3.1 of MDB and to what degree that was an original finding of MDB. In any case, it is apparent that the level of simplicity adopted by MDB in their critique has not adequately matched the fundamental nature of the physical problems MDB took up to analyze. The critique of MDB therefore forms a solid ground zero platform for further analysis and development of the evaporative force concept that one can rightfully expect to be made on physically deeper and more comprehensive grounds.

\section{Concluding remarks: new circulation driver calls for attention}

Missing from textbooks, the evaporative force emerges as a physically distinct driver of atmospheric circulation that yields observable air velocities. Its existence does not contradict or replace the existence of air motions driven by physically independent buoyancy effects, yet calls one to re-appraise the importance of the latter. The evaporative force concept provides answers to some of the key problems that are challenging the current understanding of atmospheric processes. For example, as outlined by MDB in Sect. 4, the traditional paradigm considers differential heating to be the major driver of atmospheric circulation. However, this consideration critically fails in the case of the strongest winds observed on Earth, the hurricanes, that, as is well-known (Riehl, 1950), develop along nearly isothermal surfaces. But if differential heating is not necessary for producing the strongest winds, perhaps it is not indispensable for producing moderate and weak winds either? The evaporative force concept that relates wind velocities to spatial differences in the intensity of condensation rather than heating provides a unifying explanation to both hurricanes and tornadoes as well as to stationary circulation patterns (Makarieva et al., 2008; Makarieva and Gorshkov, 2009). On a related note, according to the traditional paradigm the regions of air ascent should be associated with positive buoyancy. In contrast, observations of atmospheric updrafts indicate a wide range of positive and negative buoyances (Folkins, 2006). The evaporative force concept resolves the puzzle. Air pressure depends on two independent variables, temperature and number of air molecules in a unit volume. Consequently, there are two independent ways of making local air pressure higher than that in the neighboring area, to initiate air motion - (1) to locally warm the air (this is what the traditional paradigm of horizontal differential heating is about) and (2) to reduce the number of air molecules in the neighboring area (this is what condensation is doing in the vertical dimension). Thus, if the condensation is intense, it can make even dense cold air rise from the surface by creating a strong weight imbalance in the upper part of the air column.

But apparently the most important implication of the evaporative force that raises most interest (Chown and Gaston, 2008; Sheil and Murdiyarso, 2009) is the biotic pump of atmospheric moisture. In the stationary case condensation is more intense where evaporation is more intense, hence the evaporative force makes air move from areas with lower to areas with higher evaporation. MDB rightly point out (Sect. 3.2) that oceans are strong evaporators and could themselves "suck" air from the continents. The statement of MDB that this point was "not at all considered by MG" does not however conform to reality, as it is the central point of the biotic pump theory. In Fig. 4 of MG several cases are summarized where, depending on the available solar flux and the state of terrestrial vegetation, oceans come as stronger or weaker evaporators than the continents. Most importantly, it was necessary for natural forests with high leaf area index to appear in the course of biological evolution for evaporation from the forest canopy to exceed evaporation from the open water surface. This allowed life to invade the hitherto dry landmasses by "sucking" moist oceanic air inland as the forests marched forward from the coast. Not surprisingly, modern global circulation models built neglecting the biotic pump physics fail radically when attempting to account for the water budget of the strongest biotic pump on Earth the Amazon river basin. The amount of oceanic moisture "brought" to the Amazon river basin by the models (the modelled atmospheric moisture convergence) proves to be twice less than the actual amount empirically estimated from the value of the Amazon runoff (Marengo, 2005). It is obvious that the traditional accounts of moisture transport in the other great river basins, including Siberian and North American rivers (Makarieva et al., 2009), will similarly have to be seriously reconsidered to incorporate the major effects of 
the forest moisture pumps, the anthropogenic destruction of which is currently threatening to turn the landmasses back into primordial deserts.

Acknowledgements. We benefited greatly from the comments made during the Interactive Discussion of the paper of Meesters et al. (2009) at HESSD. We would like to express our sincere appreciation to HESS Editors for guiding the constructive and meaningful discussion and to all discussion participants for their valuable inputs.

Edited by: B. van den Hurk

\section{References}

Chown, S. L. and Gaston, K. J.: Macrophysiology for a changing world, Proc. Biol. Sci., 275, 1469-1478, 2008.

Feynman, R. P., Leighton, R. B., and Sands, M.: The Feynman Lectures on Physics, Vol. 1, Addison-Wesley, Reading, 1963.

Folkins, I.: Convective damping of buoyancy anomalies and its effect on lapse rates in the tropical lower troposphere, Atmos. Chem. Phys., 6, 1-12, 2006,

http://www.atmos-chem-phys.net/6/1/2006/.

Makarieva, A. M. and Gorshkov, V. G.: Biotic pump of atmospheric moisture as driver of the hydrological cycle on land, Hydrol. Earth Syst. Sci., 11, 1013-1033, 2007, http://www.hydrol-earth-syst-sci.net/11/1013/2007/.
Makarieva, A. M. and Gorshkov, V. G.: Condensation-induced dynamic gas fluxes in a mixture of condensable and noncondensable gases, Phys. Lett. A, 373, 2801-2804, 2009.

Makarieva, A. M., Gorshkov, V. G., and Li, B.-L.: Conservation of water cycle on land via restoration of natural closed-canopy forests: Implications for regional landscape planning, Ecol. Res., 21, 897-906, 2006.

Makarieva, A. M., Gorshkov, V. G., and Li, B.-L.: On the validity of representing hurricanes as Carnot heat engine, Atmos. Chem. Phys. Discuss., 8, 17423-17437, 2008, http://www.atmos-chem-phys-discuss.net/8/17423/2008/.

Makarieva, A. M., Gorshkov, V. G., and Li, B.-L.: Precipitation on land versus distance from the ocean: Evidence for a forest pump of atmospheric moisture, Ecol. Complexity, doi:10.1016/j.ecocom.2008.11.004, in press, 2009.

Marengo, J. A.: Characteristics and spatio-temporal variability of the Amazon River Basin Water Budget, Clim. Dynam. 24, 1122, 2005.

Meesters, A. G. C. A., Dolman, A. J., and Bruijnzeel, L. A.: Comment on "Biotic pump of atmospheric moisture as driver of the hydrological cycle on land" by A. M. Makarieva and V. G. Gorshkov, Hydrol. Earth Syst. Sci., 11, 1013-1033, 2007, Hydrol. Earth Syst. Sci., 13, 1299-1305, 2009, http://www.hydrol-earth-syst-sci.net/13/1299/2009/.

Riehl, H.: A model of hurricane formation, J. Appl. Phys. 21, 917925, 1950.

Sheil, D. and Murdiyarso, D.: How forests attract rain: an examination of a new hypothesis, Bioscience, 59, 341-347, 2009. 